\title{
CHIARITÉ
}

UNIVERSITÄTSMEDIZIN BERLIN

\section{Functional connectivity between prefrontal cortex and subgenual cingulate predicts antidepressant effects of ketamine}

Matti Gärtner, Sabine Aust Malek Bajbouj, Yan Fan, Katja Wingenfeld, Christian Otte, Isabella Heuser-Collier, Heinz Böker, Josef Hättenschwiler, Erich Seifritz, Simone Grimm, Milan Scheidegger

\section{Document type}

Postprint (accepted version)

This version is available at

https://doi.org/10.17169/refubium-31346

\section{Citation details}

Gärtner M, Aust S, Bajbouj M, Fan Y, Wingenfeld K, Otte C, et al. Functional connectivity between prefrontal cortex and subgenual cingulate predicts antidepressant effects of ketamine. European Neuropsychopharmacology. [Online] Elsevier BV; 2019;29(4): 501-508.

DOI: $10.1016 /$ j.euroneuro.2019.02.008

\section{Terms of use}

This work is licensed under a Creative Commons Attribution-NonCommercial-NoDerivatives 4.0 International license: https://creativecommons.org/licenses/by-nc-nd/4.0/ 


\section{Functional connectivity between prefrontal cortex and subgenual cingulate predicts antidepressant effects of ketamine}

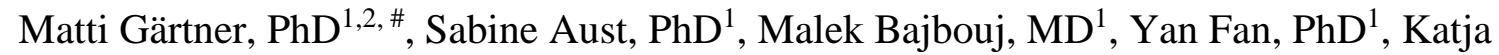
Wingenfeld, $\mathrm{PhD}^{1}$, Christian Otte, $\mathrm{MD}^{1}$, Isabella Heuser-Collier, $\mathrm{MD}^{1}$, Heinz Böker, $\mathrm{MD}^{3}$, Josef Hättenschwiler, $\mathrm{MD}^{4}$, Erich Seifritz, $\mathrm{MD}^{3}$, Simone Grimm, $\mathrm{PhD}^{1,2,3^{*}}$, Milan Scheidegger, $\mathrm{MD}^{3 *}$

\section{Affiliations:}

${ }^{1}$ Charité - Universitätsmedizin Berlin, corporate member of Freie Universität Berlin, Humboldt-Universität zu Berlin, and Berlin Institute of Health, Department of Psychiatry and Psychotherapy, Campus Benjamin Franklin, Hindenburgdamm 30, 12203 Berlin, Germany

${ }^{2}$ MSB - Medical School Berlin, Calandrellistraße 1-9, 12247 Berlin

${ }^{3}$ Department of Psychiatry, Psychotherapy and Psychosomatics, Psychiatric Hospital, University of Zurich, Lenggstrasse 31, 8032 Zurich, Switzerland

${ }^{4}$ Center for Anxiety and Depression (ZADZ), Riesbachstrasse 61, 8008 Zurich, Switzerland

* These authors contributed equally

\# Correspondence: Matti Gärtner, Charité University Medicine Berlin, Clinic for Psychiatry and Psychotherapy, Hindenburgdamm 30, 12203 Berlin, Germany. Email: matti.gaertner@charite.de, Phone: +49 (0) 30450 517778, Fax: +49 (0) 30450517942 


\begin{abstract}
Converging evidence suggests that a single sub-anesthetic dose of ketamine can produce strong and rapid antidepressant effects in patients that do not respond to standard treatment. Despite a considerable amount of research investigating ketamine's mechanisms of action, the exact neuronal targets conveying the antidepressant effects have not been identified yet. Preclinical studies suggest that molecular changes induced by ketamine bring forward large-scale network reconfigurations that might relate to ketamine's antidepressant properties. In this prospective two-site study we measured resting state fMRI in 24 depressed patients prior to, and 24 hours after a single sub-anesthetic dose of ketamine. We analyzed functional connectivity (FC) at baseline and after ketamine and focused our analysis on baseline FC and FC changes directly linked to symptom reduction in order to identify neuronal targets that predict individual clinical responses to ketamine. Our results show that FC increases after ketamine between right lateral prefrontal cortex (PFC) and subgenual anterior cingulate cortex (sgACC) are positively linked to treatment response. Furthermore, low baseline FC between these regions predicts treatment outcome. We conclude that PFC-sgACC connectivity may represent a promising biomarker with both predictive and explanatory power.
\end{abstract}




\section{Introduction}

Increasing evidence suggests that a single sub-anesthetic dose of the N-methyl-D-aspartate (NMDA) receptor antagonist ketamine has strong and rapid antidepressant properties, especially in cases of otherwise treatment-resistant depression (TRD; Berman et al., 2000; McGirr et al., 2015; Murrough et al., 2013; Zarate et al., 2006). Compared to other antidepressant treatment strategies, the most remarkable property of ketamine is the rapid onset of the response often within a few hours after initial administration (Machado-Vieira et al., 2009). Discovery of rapid acting antidepressants is crucial because the delayed onset of two or more weeks of most commonly prescribed drugs in not without serious burden for patients suffering from major depressive disorder (MDD) and bears the risk of suicidality. Despite the promising discovery of ketamine's unique antidepressant properties, the exact biomechanisms associated with ketamine's antidepressant efficacy remain largely unclear. Over the past two decades, a large number of preclinical studies have investigated ketamine's molecular mechanisms and putative antidepressant targets have been proposed. However, ketamine has a heterogeneous pharmacodynamic profile, and mechanistic evidence from patient populations is needed to directly link ketamine-induced brain changes to the improvement of clinical symptoms.

Animal models suggest that ketamine triggers a cascade of intracellular events that induce changes in synaptic plasticity (Duman et al., 2012), as well as rapid synaptogenesis in the prefrontal cortex (PFC; Li et al., 2010). These effects of ketamine on synaptic structure and function have been related to its antidepressant effects in behavioral animal models of depression (Autry et al., 2011; Li et al., 2010). Furthermore, it has been suggested that the local synaptic changes ketamine induces in the brain bring forward large-scale network reconfigurations that may relate to its antidepressant properties (Lv et al., 2016), and it is well- 
established that depression is linked to alterations in large-scale brain networks (Brakowski et al., 2017; Kaiser et al., 2016). The neuroimaging literature on how ketamine affects human brain networks is still at an early stage, and the majority of studies have been conducted in healthy volunteers. However, previous studies consistently report ketamine effects on brain regions that have also been linked to depression, such as the subgenual anterior cingulate cortex (sgACC), posterior parietal cortex (PCC), prefrontal cortex (PFC), and hippocampus (Ionescu et al., 2018). Among these regions the sgACC has been repeatedly reported. While studies in healthy volunteers reported a rapid decrease of sgACC activity during a ketamine infusion (Deakin et al., 2008), a ketamine-induced increase in activity has been described in depressed patients (Downey et al., 2016; Nugent et al., 2014). It remains an open question, though, whether these contrary findings relate to differential effects in patients and healthy volunteers, or to methodological differences between studies. In a study investigating functional connectivity (FC) changes after ketamine in healthy volunteers, Scheidegger et al. (2012) found decreased FC between the sgACC and the medial prefrontal cortex as well as within regions of the default mode network (DMN; Raichle et al., 2001) such as the pregenual anterior cingulate cortex (PACC), medial prefrontal cortex (MPFC) and posterior cingulate cortex (PCC). One of the very few studies investigating FC changes after ketamine in MDD patients found that ketamine increased global FC of the right lateral PFC, and further showed that this increase was larger in responders than in non-responders (Abdallah et al., 2017). Another recent FC study in MDD patients found that connectivity of the DMN to the insula was normalized after ketamine when compared to healthy volunteers (Evans et al., 2018). Taken together, these findings suggest that ketamine alters activity in and connectivity between brain regions involved in depression. Furthermore, the current state of research suggests that the antidepressant effects of ketamine are linked to reduced activity in brain networks related to self-referential processing, and increased activity in cognitive control networks (Ionescu et al., 
2018). However, methodological differences, and the limited number of studies directly linking ketamine-induced brain changes to the improvement of depressive symptoms, make it difficult to draw definite conclusions. On the other hand, the existing findings suggest that the investigation of large-scale connectivity networks using resting-state fMRI in humans might be particularly useful to study the antidepressant effects of ketamine.

Against this background, we measured resting state brain activity in 24 depressed patients before, and 24 hours after a single sub-anesthetic ketamine infusion in two independent psychiatric departments. Specifically, we hypothesized that FC changes after ketamine treatment between regions that have previously been linked to depression are directly associated with the improvement of depressive symptoms. In addition, we exploratively investigated whether baseline FC can be used to predict the response to the ketamine treatment.

\section{Methods}

\section{Participants}

Male and female patients diagnosed with MDD were recruited at the Department of Psychiatry and Psychotherapy, Charité - Universitätsmedizin Berlin (CHAR), and at the Department of Psychiatry, Psychotherapy and Psychosomatics, Psychiatric Hospital, University of Zurich (UZH). Patients were "treatment-resistant" being defined as two or more sufficient antidepressant treatment trials during the current episode without achieving remission. There were no restrictions regarding antidepressant medication at the time of enrolment, however, medication intake was documented (see Results section). Exclusion criteria comprised lifetime antidepressant treatment with ketamine, lifetime recreational use of ketamine, cardiovascular diseases such as hypertonia, cardiac insufficiency or myocardial infarct in the past six months, insufficiently treated anemia, hyper- or hypothyroidism, lifetime increased intracranial 
pressure or glaucoma, chronic physical diseases, in particular hepatorenal dysfunction, recent heart or head surgery, current pregnancy, as well as any relevant psychiatric or neurological comorbidity, in particular dementia, epileptic seizures, schizophrenia, psychosis, or posttraumatic stress disorder, substance abuse disorders, acute suicidality. Additional exclusion criteria regarding the scanning procedure were metallic body implants and claustrophobia. The study was carried out in accordance with the latest version of the Declaration of Helsinki and approved by the Institutional Review Board of Charité - Universitätsmedizin Berlin and the Ethics Committee Zurich. All patients gave written informed consent before participation.

\section{Study design}

All participants underwent a baseline resting state fMRI scan prior to the ketamine infusion (2 - 24 hours). After the baseline scan participants received an intravenous ketamine infusion (45 mins) of either $0.5 \mathrm{mg} / \mathrm{kg}$ of racemic ketamine (R/S, enantiomer ratio of $1: 1$, received at CHAR) or S-ketamine $(0.25 \mathrm{mg} / \mathrm{kg}$, Ketanest $\mathrm{S}$, received at $\mathrm{UZH})$. The $\mathrm{S}(+)$-isomer of ketamine is characterized by a 3-4 times higher affinity or potency at specific receptors, so that a dose reduction of $50 \%$ is recommended (Sinner and Graf, 2008). Although more studies applied racemic ketamine in previous studies, similar effect sizes have been reported for Sketamine (Singh et al., 2016), and differences with respect to antidepressant mechanisms of action are unknown. At UZH, S-ketamine is used to treat depressed patients and racemic ketamine is used at CHAR. For this study we decided not to change the clinical routine, and to investigate potential differences between substances as an exploratory endpoint. Since the antidepressant effect of ketamine is most prominent after one day (Zarate et al., 2006), the follow-up fMRI scans were scheduled 24 hours after the ketamine infusion to assess the related 
effects on neuronal network dynamics that might contribute to the understanding of its antidepressant efficacy.

\section{Psychological Assessment}

Depression severity was assessed at baseline and 24 hours after the ketamine infusion, using the Montgomery-Åsberg Depression Rating Scale (MADRS; Montgomery and Asberg, 1979; at CHAR), and the Hamilton Rating Scale for Depression (HAM-D; Hamilton, 1980; at UZH). To achieve comparability between the two different rating scales, changes in depressive symptoms were calculated as percent-change from baseline for both scales (symptom improvement $=$ baseline - follow-up $/$ baseline $* 100)$.

\section{FMRI acquisition and analyses}

Functional data were acquired on a Siemens Trio 3T (CHAR) and a Philips Achieva TX 3-T scanner (UZH) using standard echo planar imaging sequences. At CHAR functional data were collected in 8-minute runs (210 volumes) with 37 oblique axial slices of $3 \mathrm{~mm}$ (TE $=30 \mathrm{~ms}$; field of view $=192 \mathrm{~mm}, 3 \times 3 \mathrm{~mm}$ in- plane resolution, TR $2300 \mathrm{~s}$, flip angle $70^{\circ}$ ). At UZH the functional images were collected in 10 min runs (200 volumes) with 32 contiguous axial slices of $4 \mathrm{~mm}(\mathrm{TE}=35 \mathrm{~ms}$; field of view $=22 \mathrm{~cm}, 2.75 \times 2.75 \mathrm{~mm}$ in-plane resolution, $\mathrm{TR} 3000 \mathrm{~ms}$, flip angle $82^{\circ}$, and sensitivity-encoded acceleration factor $\mathrm{R}=2.0$ ). A 3-dimensional $\mathrm{T} 1$ weighted anatomical scan was obtained for structural reference at both scanning sites.

All resting state fMRI data were analyzed in Matlab (Version R2015a) using SPM12 and the CONN toolbox (Version 17c; Whitfield-Gabrieli and Nieto-Castanon, 2012). Preprocessing of the fMRI data was performed using the default preprocessing pipeline "Direct normalization to MNI-space" available in CONN. The pipeline includes motion correction (realignment and unwarping), slice-timing correction, automatic detection of artifactual scans (ART-based 
scrubbing), normalization to MNI space, and spatial smoothing (8mm). During the denoising step in CONN single-subject linear regression analyses were performed to remove the effects of head motion (12 total motion covariates: 6 motion parameters plus temporal derivatives), physiological artifacts (10 total CompCor eigenvariates: 5 each from eroded WM and CSF masks), and artifactual scans. The resulting residual BOLD time series were band-pass filtered $(0.01-0.1 \mathrm{~Hz})$.

Seed selection was based on previous literature from our group (Scheidegger et al., 2012), and on a recent review by Ionescu et al (Ionescu et al., 2018), in which convergent brain regions modulated by ketamine were identified. The following seed regions of interest (ROI: $x, y, z$, in Montreal Neurological Institute (MNI) space) were included: the sgACC (sphere at $228-5$ with $5 \mathrm{~mm}$ radius), the left and right dorsolateral PFC (sphere at \pm 403632 with $10 \mathrm{~mm}$ radius), the left and right PCC (sphere at $\pm 6-5024$ with $7 \mathrm{~mm}$ radius), and the left and right hippocampus (anatomical seeds from the FSL Harvard-Oxford Atlas).

Single subject seed-to-voxel correlation maps were calculated by extracting the residual blood oxygen level-dependent (BOLD) time course from the seed and computing Pearson's correlation coefficients between that time course and the time course of all other voxels. Correlation coefficients were converted to normally distributed z-scores using the Fisher transformation to improve the validity of second-level General Linear Model analyses.

In the statistical group analyses we focused on pre-to-post FC changes associated with the change of depressive symptoms after 24 hours. In CONN a linear regression analysis was implemented by defining the simple main effect of symptom improvement as between-subjects contrast, and time point (pre vs. post) as between-conditions contrast. Additionally, scanning 
site (CHAR versus $\mathrm{UZH}$ ) was included as covariate in the analysis. The same analysis was performed using the baseline scans only, to investigate the predictive power of baseline FC. Statistical thresholds were set to $\mathrm{p}<0.001$ (uncorrected) at the single voxel level, and to $\mathrm{p}<$ 0.05 (FDR corrected) at the cluster level. Strict Bonferroni adjustments were applied to correct for the multiple analyses conducted (7 seeds x 2 analyses types), leading to an adjusted alpha threshold of $\mathrm{p}=0.0036$. However, unadjusted results are reported as exploratory to account for the rigidity of Bonferroni adjustments, and to minimize the conduction of Type II errors. Furthermore, post-hoc correlation analyses (Pearson's correlation coefficient, two-sided) for separate study sites were performed between mean cluster values and symptom improvement to explore potential differences between the two study sites.

\section{Results}

\section{Participants}

In total 24 MD patients (14 females, mean age: 44.4 SD 11.8) fulfilled the inclusion criteria, agreed to participate, and were included in the study. At CHAR 14 patients ( 8 females, mean age: 46.0 SD 11.1) were included in the study, and at UZH 10 patients (6 females, mean age: 42.1 SD 13.0) were included. Patients at the two scanning sites did not differ in age ( $p=0.44$, t-statistic), and sex distribution ( $\mathrm{p}=0.89$, chi-square). At CHAR, patients had an average MADRS score of 26.3 (SD 5.1, range 19 - 38) at baseline, and an average MADRS score of 17.3 (SD 5.9, range 10 - 29) after 24 hours. At UZH patients had an average HAM-D score of 21.8 (SD 4.9, range 16 - 31) at baseline, and an average HAM-D score of 15.0 (SD 6.6, range 7 - 24) after 24 hours. Psychopharmacological medication, either monotherapy or augmentation / adjunctive therapies, included SSRIs ( $\mathrm{N}=7)$, SNRIs $(\mathrm{N}=7)$, tri-/tetracyclic antidepressants $(\mathrm{N}=4)$, anticonvulsants $(\mathrm{N}=3)$, atypical neuroleptics $(\mathrm{N}=9)$, benzodiazepines $(\mathrm{N}=6)$, and melatonin $(\mathrm{N}=3)$. 


\section{Clinical Results}

$92 \%$ of the patients $(22 / 24)$ showed a reduction of depressive symptoms 24 hours after the ketamine infusion. The mean symptom improvement was $33.2 \%$ (SD 20.6\%). In total $33 \%$ $(8 / 24)$ of the patients were classified as responders (i.e. a $50 \%$ reduction of symptoms) after 24 hours. No differential effects in symptom reduction $(\mathrm{p}=0.91$, $\mathrm{t}$-statistic), or number of responders ( $\mathrm{p}=0.77$, chi-square) were observed between CHAR and UZH. At CHAR the mean Symptom improvement was $33.6 \%$ (SD $20.9 \%$ ), and 5/14 patients were classified as responders. At UZH the mean Symptom improvement was $32.6 \%$ (SD 21.4 \%), and 3/10 patients were classified as responders.

\section{fMRI Results}

The whole-brain regression analysis revealed significant FC changes related to symptom reduction for the sgACC seed (see Figure 1A for the location of the seed region). All other investigated seed regions (bilateral dorsolateral PFC, bilateral PCC, and bilateral hippocampus) did not show significant FC changes related to symptom reduction. For the sgACC seed, 4 clusters (2 Bonferroni adjusted, 2 exploratory) showed that more positive FC after ketamine was positively related to symptom reduction. All 4 clusters were located in the right frontal lobe (supplementary motor area, dorsolateral PFC, anterior PFC, and orbitofrontal cortex). See Table 1 for a detailed description of all target clusters, and Figure 1B for a visualization of the clusters on the cortical surface. Post-hoc correlation analyses revealed a strong positive relationship between single subject FC change (mean value of the cluster in the anterior PFC), and Symptom improvement for both study sites (see Figure 2A).

Furthermore, the second whole-brain regression analysis conducted to investigate the relationship between baseline FC and symptom reduction revealed that baseline FC of the 
sgACC to the right lateral anterior PFC was negatively related to symptom reduction, with lower levels of baseline connectivity corresponding to higher levels of symptom reduction 24 hours after ketamine. All other investigated seed regions did not reach statistical significance after Bonferroni correction, but some exploratory findings were observed for the right PCC, left HC, and left DLPFC. See Table 2 for a detailed description of all target clusters, and Figure $1 \mathrm{C}$ for a visualization of the cluster detected for the sgACC seed. Post-hoc correlation analyses revealed a strong negative relationship between single subject baseline FC (mean value of the cluster in the anterior PFC), and Symptom improvement for both study sites (see Figure 2A). I.e. lower baseline FC between the sgACC and anterior PFC was linked higher levels of symptom reduction after ketamine.

Remarkably, an exploratory subgroup analysis in the ketamine responders (i.e. $50 \%$ symptom reduction; N=8), showed that all eight responders (100\%) had negative baseline FC values between the sgACC and the right lateral anterior PFC. Furthermore, seven out of the eight responders $(88 \%)$ showed increases of FC between these regions after the ketamine treatment (see Figure 2 A \& B).

\section{Discussion}

In this study we investigated whether FC in large-scale brain networks is linked to the antidepressant properties of ketamine in depressed patients. Our main results show that increased connectivity after ketamine treatment between the sgACC and the right lateral PFC is linked to higher levels of symptom reduction. Furthermore, our results show that low baseline connectivity before ketamine treatment between the above regions is linked to higher levels of reduction of depressive symptoms. 
Accumulating evidence indicates important roles of the sgACC in regulating stress-related affective responses and negative affect states (Drevets et al., 2008; Shao et al., 2018), and it is well-established that the sgACC is overactive in depressed patients (Drevets et al., 2008; Mayberg et al., 1999). Furthermore, it has been shown that sgACC activity and connectivity is normalized after SSRI treatment (Mayberg et al., 2000; McCabe and Mishor, 2011; Olbrich et al., 2014). Notably, deep brain stimulation of the sgACC was also found to result in an antidepressant effect (Mayberg et al., 2005), and the antidepressant efficacy of transcranial magnetic stimulation (TMS) stimulation at specific loci within the DLPFC was strongly correlated with functional connectivity between the DLPFC site and the sgACC (Fox et al., 2012). Our findings strongly support this line of evidence, by establishing a link between the rapid antidepressant response to ketamine and increased functional connectivity between these regions. The right lateral PFC has been implicated in executive function and cognitive control (Goldman-Rakic, 1995; Koechlin et al., 1999), and increased activity in the sgACC in combination with decreased activity in the right lateral PFC has been linked to transient and chronic negative mood (Mayberg et al., 1999). Taken together these findings suggest that higher lateral PFC-sgACC connectivity might be linked to an enhanced prefrontal capacity to (down)regulate the processing of negative emotions in the sgACC. Our findings thus support the general notion that the central executive network plays a critical role in modulating emotional processing in the $\operatorname{sgACC}$, and that ketamine specifically affects this neurocircuit to restore more adaptive levels of mood regulation. Further evidence for this assumption comes from a recent TMS study, showing that FC between the stimulated rTMS site (i.e. a region in the lateral PFC) and the sgACC predicts the antidepressant response to the TMS treatment (Weigand et al., 2018). 
The assumption of enhanced prefrontal control after ketamine is in line with molecular studies in animal models showing that ketamine induces rapid synaptogenesis in the PFC (Duman et al., 2016). The rapid increase in neuronal excitability in the PFC after ketamine administration is likely to affect not only synaptic plasticity but also large-scale functional connectivity. Investigating global brain connectivity (GBC) in MDD patients Abdallah et al (2017) found an increased GBC in the lateral PFC after ketamine that was stronger in treatment responders. Our study extends this line of empirical evidence by pointing out a linear relationship between lateral PFC-sgACC connectivity and clinical response to ketamine. Increased capacity for adaptive dynamics in neurocircuits for mood regulation might thus underly ketamine-induced clinical recovery (Lehmann et al., 2016; Scheidegger et al., 2016). Ketamine's metabolism in the brain suggests that the antidepressant effects after 24 hours are due to secondary processes (Duman et al., 2012). An interesting question is whether the acute effects and network modulations induced by ketamine are linked to the antidepressant response and sustained adaptive changes in brain dynamics. To that, future studies with multistage imaging protocols for the dynamic investigation of the biomechanisms of rapid-acting antidepressants need to be developed to allow for monitoring of the individual states of a multiphasic drug response (Walter et al., 2014). Although ketamine-induced changes in neuronal connectivity and network function might be transient and heterogenous, they may nevertheless increase emotion regulation capacities and open plasticity windows for psychotherapeutic interventions (Wilkinson et al., 2017).

Previous FC studies have not identified sgACC-PFC changes after ketamine. This might be due to methodological differences for instance in acquisition protocols and drug administration. Furthermore, it must be noted that we also do not find absolute pre-to-post FC changes between these regions. Rather, we identified a specific linear relationship between baseline FC, FC 
change, and the antidepressant response, with potential clinical relevance. Since a considerable proportion of our patients already had relatively high FC between sgACC and PFC at baseline and did not show further connectivity increases or benefits from the treatment, absolute pre-topost changes did not reach thresholds for statistical significance. This supports our methodological approach to directly investigate the link between FC and symptom reduction in the whole brain analysis.

From a clinical perspective, reductions in depressive symptoms and the proportion of treatment response was comparably lower than in other clinical trials with ketamine. Partly, this could be explained by the fact that a large part of our patients was recruited from wards specialized in the treatment of chronic and severe affective disorders. They received ketamine as an alternative to electroconvulsive therapy and had a relatively high degree of treatment resistance. Thus, the clinical characteristics of our sample might differentiate from those of other ketamine trials. Furthermore, most of the studies so far have been conducted with racemic ketamine and some of our patients received S-ketamine. There is some controversy about antidepressant efficacy of the S- vs. R-enantiomers (Andrade, 2017). However, good effect sizes have also been reported for S-ketamine (Singh et al., 2016).

It must be acknowledged that the majority of patients in this study were part of a naturalistic clinical trial conducted at CHAR that did not include a placebo condition. As the primary endpoint of this study was to link FC changes after ketamine to the improvement of clinical symptoms, we argue that conclusions can be drawn from this study even without applying a placebo condition. We further argue that it can be reasonably assumed that brain changes corresponding to the rapid improvement of depressive symptoms 24 hours after ketamine in severely depressed patients can be linked to the effects of ketamine. Furthermore, ketamine has 
relatively strong, acute psychotomimetic effects, which puts into question whether a placebo condition outweighs the benefits of a naturalistic study design.

In conclusion, the reported close association of sgACC-PFC functional connectivity changes with ketamine's antidepressant response, and converging evidence from other treatment modalities (e.g. TMS, has the potential to serve as an imaging biomarker to predict whether a patient will respond to ketamine treatment. However, these findings must be confirmed in large independent patient samples. Furthermore, additional scanning time points would be of interest, e.g. to investigate how long ketamine-induced functional connectivity changes persist, and to identify optimal time points for repeated ketamine administration. 


\section{References}

Abdallah, C.G., Averill, L.A., Collins, K.A., Geha, P., Schwartz, J., Averill, C., DeWilde, K.E., Wong, E., Anticevic, A., Tang, C.Y., Iosifescu, D.V., Charney, D.S., Murrough, J.W., 2017. Ketamine Treatment and Global Brain Connectivity in Major Depression.

Neuropsychopharmacology 42, 1210-1219.

Andrade, C., 2017. Ketamine for Depression, 3: Does Chirality Matter? J Clin Psychiatry 78, e674-e677.

Autry, A.E., Adachi, M., Nosyreva, E., Na, E.S., Los, M.F., Cheng, P.F., Kavalali, E.T., Monteggia, L.M., 2011. NMDA receptor blockade at rest triggers rapid behavioural antidepressant responses. Nature 475, 91-95.

Berman, R.M., Cappiello, A., Anand, A., Oren, D.A., Heninger, G.R., Charney, D.S., Krystal, J.H., 2000. Antidepressant effects of ketamine in depressed patients. Biol Psychiatry $47,351-354$.

Brakowski, J., Spinelli, S., Dorig, N., Bosch, O.G., Manoliu, A., Holtforth, M.G., Seifritz, E., 2017. Resting state brain network function in major depression - Depression symptomatology, antidepressant treatment effects, future research. Journal of Psychiatric Research 92, 147-159.

Deakin, J.F., Lees, J., McKie, S., Hallak, J.E., Williams, S.R., Dursun, S.M., 2008. Glutamate and the neural basis of the subjective effects of ketamine: a pharmaco-magnetic resonance imaging study. Arch Gen Psychiatry 65, 154-164.

Downey, D., Dutta, A., McKie, S., Dawson, G.R., Dourish, C.T., Craig, K., Smith, M.A., McCarthy, D.J., Harmer, C.J., Goodwin, G.M., Williams, S., Deakin, J.F., 2016. Comparing the actions of lanicemine and ketamine in depression: key role of the anterior cingulate. Eur Neuropsychopharmacol 26, 994-1003. 
Drevets, W.C., Savitz, J., Trimble, M., 2008. The subgenual anterior cingulate cortex in mood disorders. CNS Spectr 13, 663-681.

Duman, R.S., Aghajanian, G.K., Sanacora, G., Krystal, J.H., 2016. Synaptic plasticity and depression: new insights from stress and rapid-acting antidepressants. Nat Med 22, 238-249.

Duman, R.S., Li, N., Liu, R.J., Duric, V., Aghajanian, G., 2012. Signaling pathways underlying the rapid antidepressant actions of ketamine. Neuropharmacology 62, 35-41.

Evans, J.W., Szczepanik, J., Brutsche, N., Park, L.T., Nugent, A.C., Zarate, C.A., Jr., 2018. Default Mode Connectivity in Major Depressive Disorder Measured Up to 10 Days After Ketamine Administration. Biol Psychiatry.

Fox, M.D., Buckner, R.L., White, M.P., Greicius, M.D., Pascual-Leone, A., 2012. Efficacy of transcranial magnetic stimulation targets for depression is related to intrinsic functional connectivity with the subgenual cingulate. Biol Psychiatry 72, 595-603.

Goldman-Rakic, P.S., 1995. Architecture of the prefrontal cortex and the central executive. Ann N Y Acad Sci 769, 71-83.

Hamilton, M., 1980. Rating Depressive Patients. J Clin Psychiat 41, 21-24. Ionescu, D.F., Felicione, J.M., Gosai, A., Cusin, C., Shin, P., Shapero, B.G., Deckersbach, T., 2018. Ketamine-Associated Brain Changes: A Review of the Neuroimaging Literature. Harv Rev Psychiatry.

Kaiser, R.H., Whitfield-Gabrieli, S., Dillon, D.G., Goer, F., Beltzer, M., Minkel, J., Smoski, M., Dichter, G., Pizzagalli, D.A., 2016. Dynamic Resting-State Functional Connectivity in Major Depression. Neuropsychopharmacology 41, 1822-1830.

Koechlin, E., Basso, G., Pietrini, P., Panzer, S., Grafman, J., 1999. The role of the anterior prefrontal cortex in human cognition. Nature 399, 148-151.

Lehmann, M., Seifritz, E., Henning, A., Walter, M., Boker, H., Scheidegger, M., Grimm, S., 2016. Differential effects of rumination and distraction on ketamine induced modulation of 
resting state functional connectivity and reactivity of regions within the default-mode network. Soc Cogn Affect Neur 11, 1227-1235.

Li, N., Lee, B., Liu, R.J., Banasr, M., Dwyer, J.M., Iwata, M., Li, X.Y., Aghajanian, G., Duman, R.S., 2010. mTOR-dependent synapse formation underlies the rapid antidepressant effects of NMDA antagonists. Science 329, 959-964.

Lv, Q., Yang, L., Li, G., Wang, Z., Shen, Z., Yu, W., Jiang, Q., Hou, B., Pu, J., Hu, H., 2016. Large-Scale Persistent Network Reconfiguration Induced by Ketamine in Anesthetized Monkeys: Relevance to Mood Disorders. Biol Psychiatry 79, 765-775.

Machado-Vieira, R., Salvadore, G., Diazgranados, N., Zarate, C.A., Jr., 2009. Ketamine and the next generation of antidepressants with a rapid onset of action. Pharmacol Ther 123, 143150.

Mayberg, H.S., Brannan, S.K., Tekell, J.L., Silva, J.A., Mahurin, R.K., McGinnis, S., Jerabek, P.A., 2000. Regional metabolic effects of fluoxetine in major depression: serial changes and relationship to clinical response. Biol Psychiatry 48, 830-843.

Mayberg, H.S., Liotti, M., Brannan, S.K., McGinnis, S., Mahurin, R.K., Jerabek, P.A., Silva, J.A., Tekell, J.L., Martin, C.C., Lancaster, J.L., Fox, P.T., 1999. Reciprocal limbic-cortical function and negative mood: converging PET findings in depression and normal sadness. Am J Psychiatry 156, 675-682.

Mayberg, H.S., Lozano, A.M., Voon, V., McNeely, H.E., Seminowicz, D., Hamani, C., Schwalb, J.M., Kennedy, S.H., 2005. Deep brain stimulation for treatment-resistant depression. Neuron 45, 651-660.

McCabe, C., Mishor, Z., 2011. Antidepressant medications reduce subcortical-cortical resting-state functional connectivity in healthy volunteers. Neuroimage 57, 1317-1323.

McGirr, A., Berlim, M.T., Bond, D.J., Fleck, M.P., Yatham, L.N., Lam, R.W., 2015. A systematic review and meta-analysis of randomized, double-blind, placebo-controlled trials 
of ketamine in the rapid treatment of major depressive episodes. Psychological Medicine 45, 693-704.

Montgomery, S.A., Asberg, M., 1979. A New Depression Scale Designed to be Sensitive to Change.

Murrough, J.W., Iosifescu, D.V., Chang, L.C., Al Jurdi, R.K., Green, C.E., Perez, A.M., Iqbal, S., Pillemer, S., Foulkes, A., Shah, A., Charney, D.S., Mathew, S.J., 2013. Antidepressant efficacy of ketamine in treatment-resistant major depression: a two-site randomized controlled trial. Am J Psychiatry 170, 1134-1142.

Nugent, A.C., Diazgranados, N., Carlson, P.J., Ibrahim, L., Luckenbaugh, D.A., Brutsche, N., Herscovitch, P., Drevets, W.C., Zarate, C.A., Jr., 2014. Neural correlates of rapid antidepressant response to ketamine in bipolar disorder. Bipolar Disord 16, 119-128.

Olbrich, S., Trankner, A., Chittka, T., Hegerl, U., Schonknecht, P., 2014. Functional connectivity in major depression: increased phase synchronization between frontal cortical EEG-source estimates. Psychiatry Res 222, 91-99.

Raichle, M.E., MacLeod, A.M., Snyder, A.Z., Powers, W.J., Gusnard, D.A., Shulman, G.L., 2001. A default mode of brain function. P Natl Acad Sci USA 98, 676-682.

Scheidegger, M., Henning, A., Walter, M., Lehmann, M., Kraehenmann, R., Boeker, H., Seifritz, E., Grimm, S., 2016. Ketamine administration reduces amygdalo-hippocampal reactivity to emotional stimulation. Human Brain Mapping 37, 1941-1952.

Scheidegger, M., Walter, M., Lehmann, M., Metzger, C., Grimm, S., Boeker, H., Boesiger, P., Henning, A., Seifritz, E., 2012. Ketamine Decreases Resting State Functional Network Connectivity in Healthy Subjects: Implications for Antidepressant Drug Action. PLoS One 7.

Shao, R., Lau, W.K.W., Leung, M.K., Lee, T.M.C., 2018. Subgenual anterior cingulateinsula resting-state connectivity as a neural correlate to trait and state stress resilience. Brain Cogn 124, 73-81. 
Singh, J.B., Fedgchin, M., Daly, E., Xi, L.W., Melman, C., De Bruecker, G., Tadic, A., Sienaert, P., Wiegand, F., Manji, H., Drevets, W.C., Van Nueten, L., 2016. Intravenous Esketamine in Adult Treatment-Resistant Depression: A Double-Blind, DoubleRandomization, Placebo-Controlled Study. Biol Psychiat 80, 424-431.

Sinner, B., Graf, B.M., 2008. Ketamine. Handb Exp Pharmacol, 313-333.

Walter, M., Li, S., Demenescu, L.R., 2014. Multistage drug effects of ketamine in the treatment of major depression. Eur Arch Psychiatry Clin Neurosci 264 Suppl 1, S55-65.

Weigand, A., Horn, A., Caballero, R., Cooke, D., Stern, A.P., Taylor, S.F., Press, D., Pascual-Leone, A., Fox, M.D., 2018. Prospective Validation That Subgenual Connectivity Predicts Antidepressant Efficacy of Transcranial Magnetic Stimulation Sites. Biol Psychiatry $84,28-37$.

Whitfield-Gabrieli, S., Nieto-Castanon, A., 2012. Conn: a functional connectivity toolbox for correlated and anticorrelated brain networks. Brain Connect 2, 125-141.

Wilkinson, S.T., Wright, D., Fasula, M.K., Fenton, L., Griepp, M., Ostroff, R.B., Sanacora, G., 2017. Cognitive Behavior Therapy May Sustain Antidepressant Effects of Intravenous Ketamine in Treatment-Resistant Depression. Psychother Psychosom 86, 162-167.

Zarate, C.A., Jr., Singh, J.B., Carlson, P.J., Brutsche, N.E., Ameli, R., Luckenbaugh, D.A., Charney, D.S., Manji, H.K., 2006. A randomized trial of an N-methyl-D-aspartate antagonist in treatment-resistant major depression. Arch Gen Psychiatry 63, 856-864. 


\section{Figures}

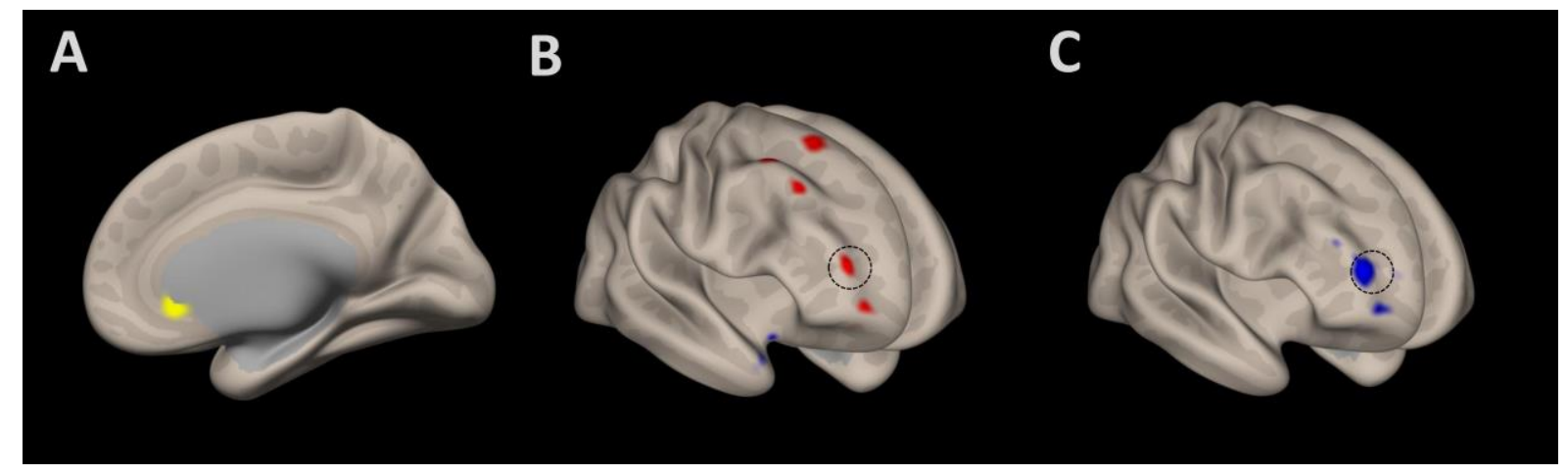

Figure 1: Resting state functional connectivity (FC) related to the reduction of depressive symptoms 24 hours after ketamine. (A) Yellow color marks the seed region in the subgenual anterior cingulate cortex ( $\operatorname{sgACC}$ ) that was used for the seed-to-voxel analysis. (B) Red color marks regions whose $\mathbf{F C}$ change to the sgACC $24 \mathrm{~h}$ after ketamine is positively related to symptom reduction (higher connectivity after ketamine = high symptom reduction). (C) Blue color marks regions whose baseline $\mathbf{F C}$ to the sgACC is negatively related to symptom reduction (low baseline connectivity $=$ high symptom reduction). Statistical thresholds for (B) and $(\mathrm{C})$ were $\mathrm{p}<0.001$ at the voxel level, and $\mathrm{p}<0.05$ (FDR corrected) at the cluster level. 


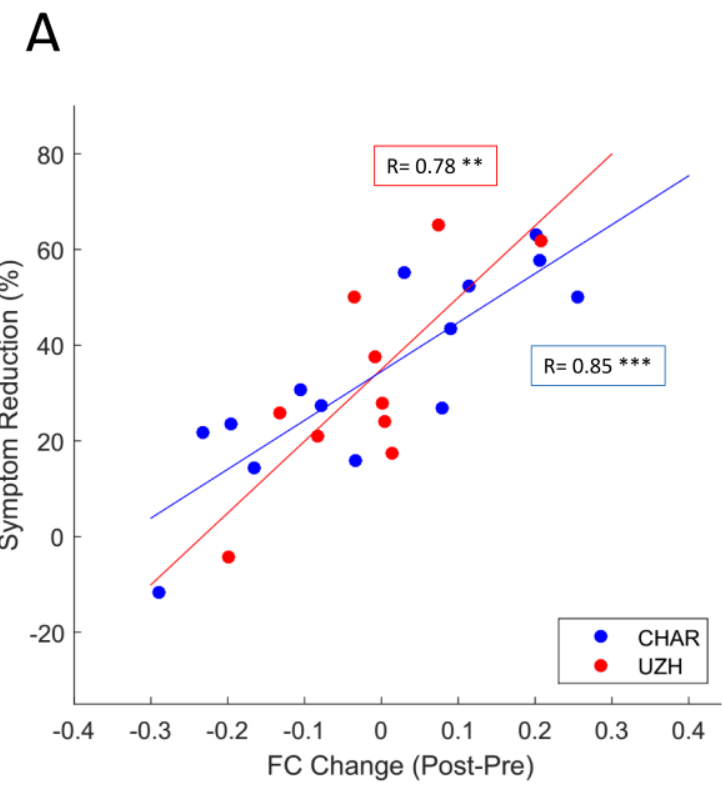

\section{B}

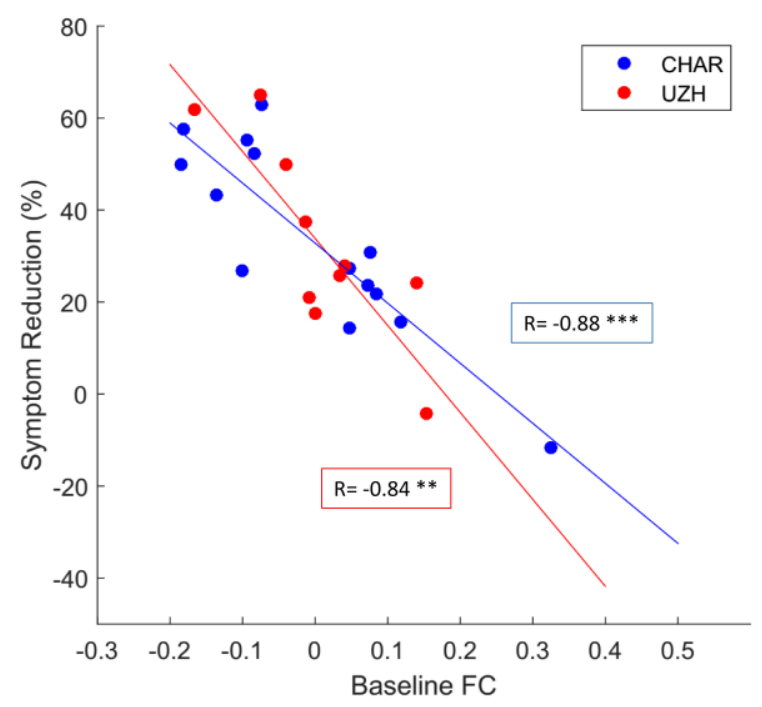

Figure 2: Post-hoc correlation analyses of resting state functional connectivity (FC) related to the reduction of depressive symptoms after ketamine at separate study sites. (A) Relationship between FC change (sgACC - rPFC, cp. Figure 1B marked region) $24 \mathrm{~h}$ after ketamine and symptom reduction. (B) Relationship between baseline FC (sgACC - rPFC, cp. Figure 1C marked region) and symptom reduction. The R-value depicts Pearson's Correlation Coefficient $(* * *$ the corresponding P-value is $<0.001)$. Red and blue dots depict patients from the two different scanning sites (UZH and CHAR). 


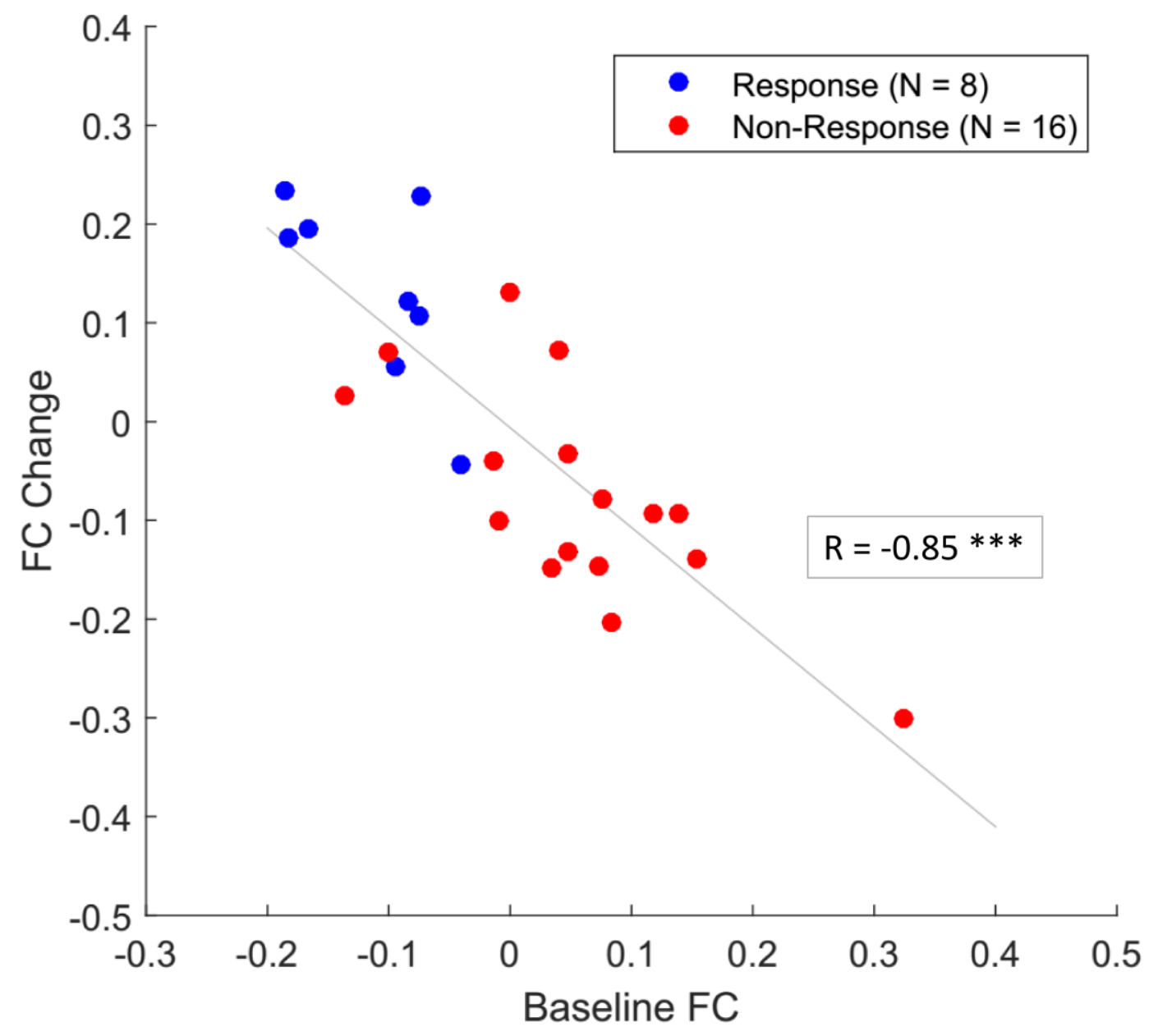

Supplementary Figure S1: Relationship between sgACC-rPFC baseline FC and FC change $24 \mathrm{~h}$ after ketamine. The R-value depicts Pearson's Correlation Coefficient (*** the corresponding P-value is < 0.001). Blue dots depict patients that responded to the ketamine treatment (symptom reduction $>=50 \%$ ). Red dots depict patients that did not respond to the treatment (symptom reduction $<50 \%$ ). 
Tables

Table 1: Seeds and regions showing FC changes related to symptom reduction

\begin{tabular}{|c|c|c|c|c|c|}
\hline Seed & Region & Direction & Coordinates & Cluster size & Size p-FDR \\
\hline sgACC & $\begin{array}{c}\text { Right MFG/SFG, } \\
\text { SMA, BA6 }\end{array}$ & $\uparrow$ & 202262 & 204 & 0.0002 \\
\hline $\begin{array}{c}\text { Right DLPFC } \\
\text { BA8, BA9 }\end{array}$ & $\uparrow$ & 423842 & 138 & 0.0015 \\
\hline $\begin{array}{c}\text { Right anterior PFC, } \\
\text { BA10, BA46 }\end{array}$ & $\uparrow$ & 365410 & 87 & 0.012 \\
\hline & $\begin{array}{c}\text { Right OFC/FP, } \\
\text { BA10 }\end{array}$ & $\uparrow$ & $3260-2$ & 74 & 0.018 \\
\hline
\end{tabular}

sgACC - subgenual anterior cingulate cortex; MFG - medial frontal gyrus; SFG - superior frontal gyrus; SMA - supplementary motor area; BA - Brodmann area; DLPFC dorsolateral prefrontal cortex; PFC - prefrontal cortex; OFC - orbitofrontal cortex; FP frontopolar cortex 
Table 2: Seeds and regions showing baseline FC related to symptom reduction

\begin{tabular}{|c|c|c|c|c|c|}
\hline Seed & Region & Direction & Coordinates & Cluster size & Size p-FDR \\
\hline sgACC & Right anterior PFC & $\downarrow$ & 34608 & 332 & $<0.0001$ \\
\hline Right PCC & Right IPL & $\uparrow$ & $54-5024$ & 106 & 0.032 \\
\hline & Left PCC & $\uparrow$ & $-6-4026$ & 90 & 0.033 \\
\hline Left HC & Right LOC & $\uparrow$ & $42-8014$ & 111 & 0.042 \\
\hline $\begin{array}{c}\text { Left } \\
\text { DLPFC }\end{array}$ & Caudate & $\downarrow$ & -482 & 154 & 0.0079 \\
\hline
\end{tabular}

sgACC - subgenual anterior cingulate cortex; PFC - prefrontal cortex; PCC - posterior parietal cortex; IPL - inferior parietal lobule; HC - hippocampus; LOC - lateral occipital cortex; DLPFC - dorsolateral prefrontal cortex 